\title{
The specificities of the nature of neutrinos
}

\author{
V. P. Efrosinin \\ Institute for Nuclear Research, RAS, Moscow, Russia
}

Email address:

vladimir.efrosinin@mail.ru

To cite this article:

V. P. Efrosinin. The Specificities of the Nature of Neutrinos. American Journal of Modern Physics. Vol. 3, No. 6, 2014 , pp. $245-246$. doi: 110.11648/j.ajmp.20140306.17

Abstract: The paper deals with the problem of lepton flavor and neutrino oscillation. The oscillation property concerns only the light neutrino. The discussion about the mixing of the light and so-called heavy neutrinos seems to be doubtful.

Keywords: Lepton Flavor, Neutrino Oscillation, the Standard Model, Majoran and Dirac particles

The charged leptons and quarks are described by virtue of the Dirac equation. The same equation is valid for description of the charge-neutral particles with a spin-1/2 under condition of identity and non-identity of particles. In case of truly neutrally charged particles with spin-1/2 the Dirac equation is replaced by the Majoran equation. The structure of the Standard model $[1,2,3]$ is such that after a spontaneous symmetry alteration neutrino remains the massless particle. The standard model is based on $S U(2)_{L} \times U(1)_{Y}$ gage group. However it captures only the gauge bosons of the model but the higgs and fermion sectors remain arbitrary. Accordingly, the modifications of the Standard model, where the neutrinos become already the massive particles are divided into three classes: 1) The models with additional leptons; 2) the models with extended Higgs sector; 3) the models including both new leptons and new Higgs bosons. If a neutrino is the Majoran particle, then in some processes with neutrinos the conservation low of the lepton flavour (LF) will be violated.

The low had to explain the reasons, when by interacting of neutrinos with substance the reactions [4]

$$
v_{l}+(A, Z) \rightarrow l+(A, Z+1)
$$

where $l=e^{-}, \mu^{-}, \tau$, were admissible, while the reactions

$$
\bar{v}_{l}+(A, Z) \rightarrow l+(A, Z+1)
$$

turned out to be forbidden. By imputing to the leptons ( $v_{l}$ and $l$ ) (LF) $L$, which is equal to +1 , and to the antileptons $\left(\bar{v}_{l}\right.$ and $\bar{l}$ ) $L=-1$, it is also assumed that

$$
\sum_{i} L_{i}=\text { const } .
$$

At the same time $v_{l}$ and $\bar{v}_{l}$ have represented respectively a particle and antiparticle, each of which could be in two states with different values of helicity. According to this approach the neutrino can be treated as the Dirac particle. But such a treatment of reality is not a unique possible. It is fair to assume that the neutrino is a truly neutral particle, that is $v_{l}$ and $\bar{v}_{l}$ - are simply two states of one particle with different values of helicity. Leptonic number of such neutrinos is equal to zero and we are speaking about them like about particles with Majoran nature. If the neutrino has mass the question arises whether it is a pure own weak state described by the standard model. Or maybe it is a superposition of other neutrino states. If neutrinos have mixed states, then the neutrino oscillation is possible. The difference between the Majoran and Dirac particles is due to their transformation properties under the charge conjugation. Consequently, the interaction of the Majoran neutrinos can not be the an eigenstate of only one charge conjugation operator.

The case in hand is light neutrinos. In our estimation [5] the neutrino mass $\leq 0.05 \mathrm{eV}$., while the electron mass is equal to $\sim 0.511 \mathrm{MeV}$. Hence the difference in properties of these leptons is significantly connected with a difference of the masses. Infinitesimal neutrino mass leads to the instability of the quantum gyroscope with respect to the varying of the helicity, appearance of the surprising oscillation property and to the mass changing. Hence the question that has to be answered is whether this process has a random character especially of the results of the research are 
treated in framework of cosmology problems.

For checking purposes of the oscillation of the neutrino it is reasonable to use simultaneously at least two methods of the parameterization of the mixing matrix.

We use the Cabibbo-Kobayashi-Maskawa matrix for three generations of the leptons, when the neutrinos are the Dirac fermions [6]

$$
\left(\begin{array}{ccc}
c_{12} c_{13} & s_{12} c_{13} & s_{13} e^{-i \delta} \\
-s_{12} c_{23}-c_{12} s_{23} s_{13} e^{i \delta} & c_{12} c_{23}-s_{12} s_{23} s_{13} e^{i \delta} & s_{23} c_{13} \\
s_{12} s_{23}-c_{12} c_{23} s_{13} e^{i \delta} & -c_{12} s_{23}-s_{12} c_{23} s_{13} e^{i \delta} & c_{23} c_{13}
\end{array}\right),
$$

where $c_{i j}=\cos \theta_{i j}, s_{i j}=\sin \theta_{i j}$. In other parameterization

$$
\left(\begin{array}{ccc}
C_{1} & -S_{1} C_{3} & S_{1} S_{3} \\
S_{1} C_{2} & C_{1} C_{2} C_{3}-S_{2} S_{3} e^{i \delta} & C_{1} C_{2} S_{2}+S_{2} C_{3} e^{i \delta} \\
S_{1} C_{2} & C_{1} S_{2} C_{3}+C_{2} S_{3} e^{i \delta} & C_{1} S_{2} S_{3}-C_{2} C_{3} e^{i \delta}
\end{array}\right)
$$

where $C_{i}=\cos \theta_{i}, S_{i}=\sin \theta_{i}, \quad i=1,2,3$. Thus, by mixing of three types of neutrinos there are three mixing angels $\left(\theta_{12}, \theta_{23}, \theta_{13}\right)$ and one CP-violation phase $\delta$. For Majoran neutrino fields it is needed to take into account in matrix (4) additionally the dependence of two Majoran phases $\chi_{1}, \chi_{2}$. To accomplish this, the matrix (4) must be multiplied by the following matrix

$$
\left(\begin{array}{ccc}
e^{-i \chi_{1}} & 0 & 0 \\
0 & e^{-i \chi_{2}} & 0 \\
0 & 0 & 1
\end{array}\right)
$$

The greatest part of the mass of the Universe concentrates not in ordinary baryonic matter, in atoms and electrons. This conclusion receives sufficient support from the observations of the anisotropy of the relic radiation. This matter is dark in a sense that it weakly couples with a radiation, since we don't observe it. It means that these particles must be charge-neutral. The study of the dynamics of galactic clusters suggests that the dark matter particles must be cold, their speeds are considerably nonrelativistic. High-mass particles may remain up to the present moment, if they possess the conserved additive and multiplicative quantum number. If there is some corresponding to this number nonzero chemical potential, then after all antiparticles (or particles) annihilate, some of the particles (or antiparticle) must remain. But even when for these particles there exist nonzero chemical potentials, such that the initial densities of the particles' and antiparticles' number are equal, if they can annihilate only with their antiparticles, then as their densities of the numbers become sufficiently small, the collision frequency at the end becomes too small to allow further reduction of the density.

It is believed that a notable contribution to the total energy density in a modern Universe (about 20\%) is made by a dark matter, which consists of new high-mass particles missing in the Standard model. The most acceptable alternate for the research is a cold dark matter. These particles must be nonrelativistic, hardly interact with each other (or at least interact but very weakly) and do not interact with photons. So that the gas in galaxies of dark matter particles can not cool off by emitting photons like baryons. It is natural to assume that the dark matter particles $\mathrm{X}$ were in a thermodynamic equilibrium with ordinary matter in the early Universe. At some point these particles have lost equilibrium and since they spread freely. If the corresponding temperature $T_{f}$ sufficiently less then the mass of the dark matter particle $M_{x}$, then these particles rift being nonrelativistic. In this case one says about the cold dark matter. Otherwise if $T_{f} \geq M_{x}$ one considers two cases: $M_{x} \leq 1 \mathrm{eV}$ and $M_{x} \geq 1 \mathrm{eV}$. The first case corresponds to the hot dark matter and is realized for neutrinos.

Our discussion in the present paper allows us to draw some conclusions. The oscillation property concerns only the light neutrino. The discussion about the mixing of the light and so-called heavy neutrinos seems to be doubtful. The attempts to find theoretical relationships between the masses of the light and heavy neutrino seem to be doubtful as well. The mass difference is too high. The oscillation property of neutrinos has most likely the mechanical reason. The particles of the hot dark matter (e.g. neutrino of the Standard model) can be only a small part of the dark matter.

At the present time the search of the heavy neutrinos $N$ in $K$-meson decay $K^{+} \rightarrow \mu^{+} N$ is in progress. The measure of the missing energy at birth of the unique muon $\mu^{+}$is also of interest.

\section{References}

[1] S.L. Glahow. Nuclear Physics, 1961, Vol. 22, Pp. 579-588.

[2] S. Weinberg. Phys. Rev. Lett., 1967, Vol. 19, Pp. 1264-1266.

[3] A. Salam, 1969, Proc. Of the $8^{\text {th }}$ Nobel Symposium on Elementary Particle Theory.

[4] O.M. Bojarkin, G.G. Bojarkina. Physics of particles, 2013 (in Russian).

[5] V.P. Efrosinin, Nuclear Physics, 2010, Vol. 73, No 6, Pp. 975 (in Russian).

[6] Y.V. Gaponov, V.V. Khruschev, S.V. Semenov, The Nuclear Physics, 2008, Vol. 71, 163-171 (in Russian). 